\title{
Lymph Node Positivity in One-Step Nucleic Acid Amplification is a Prognostic Factor for Postoperative Cancer Recurrence in Patients with Stage II Colorectal Cancer: A Prospective, Multicenter Study
}

Michio Itabashi, $\mathrm{MD}, \mathrm{PhD}^{1}$, Hirofumi Yamamoto, $\mathrm{MD}, \mathrm{PhD}^{2}$, Naohiro Tomita, $\mathrm{MD}, \mathbf{P h D}^{3}$, Masafumi Inomata, $\mathrm{MD}, \mathrm{PhD}^{4}$, Kohei Murata, $\mathrm{MD}, \mathrm{PhD}^{5}$, Shigeoki Hayashi, $\mathrm{MD}, \mathrm{PhD}^{6}$, Yasuhiro Miyake, MD, $\mathrm{PhD}^{7}$, Seiji Igarashi, $\mathrm{MD}, \mathrm{PhD}^{8}$, Takeshi Kato, $\mathrm{MD}$, $\mathrm{PhD}^{9}$, Shingo Noura, $\mathrm{MD} \mathrm{PhD}^{10}$, Tomohisa Furuhata, $\mathbf{M D}, \mathbf{P h D}^{11}$, Heita Ozawa, $\mathbf{M D}, \mathbf{P h D}^{12}$, Ichiro Takemasa, $\mathbf{M D}, \mathbf{P h D}^{13}$, Masayoshi Yasui, MD, $\mathbf{P h D}^{14}$, Hiroshi Takeyama, $\mathbf{M D}, \mathbf{P h D}^{15}$, Shu Okamura, $\mathbf{M D}, \mathbf{P h D}^{16}$, Yuko Ohno, $\mathbf{P h D}^{17}$, and Nariaki Matsuura, $\mathrm{MD}, \mathbf{P h D}^{18}$

${ }^{1}$ Institute of Gastroenterology, Tokyo Women's Medical University, Shinjuku, Japan; ${ }^{2}$ Department of Molecular Pathology, Division of Health Sciences, Graduate School of Medicine, Osaka University, Suita, Japan; ${ }^{3}$ Division of Lower GI Surgery, Department of Surgery, Hyogo College of Medicine, Nishinomiya, Japan; ${ }^{4}$ Department of Gastroenterological and Pediatric Surgery, Faculty of Medicine, Oita University, Oita, Japan; ${ }^{5}$ Department of Surgery, Kansai Rosai Hospital, Amagasaki, Japan; ${ }^{6}$ Department of Digestive Surgery, Nihon University Hospital, Chiyoda, Japan; ${ }^{7}$ Department of Surgery, Osaka Minato Central Hospital, Osaka, Japan; ${ }^{8}$ Division of Pathology, Tsuboi Cancer Center Hospital, Koriyama, Japan; ${ }^{9}$ Department of Colorectal Surgery, National Hospital Organization Osaka National Hospital, Osaka, Japan; ${ }^{10}$ Department of Surgery, Osaka Rosai Hospital, Sakai, Japan; ${ }^{11}$ Division of Gastroenterological and General Surgery, St. Marianna University Toyoko Hospital, Kawasaki, Japan; ${ }^{12}$ Department of Colorectal Surgery, Tochigi Cancer Center, Utsunomiya, Japan; ${ }^{13}$ Department of Surgery, Surgical Oncology and Science, Sapporo Medical University, Sapporo, Japan; ${ }^{14}$ Department of Gastroenterological Surgery, Osaka International Cancer Institute, Osaka, Japan; ${ }^{15}$ Department of Surgery, Minoh City Hospital, Minoh, Japan; ${ }^{16}$ Department of Surgery, Suita Municipal Hospital, Suita, Japan;

${ }^{17}$ Department of Mathematical Health Science, Graduate School of Medicine, Osaka University, Suita, Japan; ${ }^{18}$ Osaka International Cancer Institute, Osaka, Japan

\section{ABSTRACT}

Background. For colorectal cancer (CRC) patients, the standard histological lymph node (LN) evaluation has low sensitivity. Our previously developed one-step nucleic acid amplification $\left(\mathrm{OSNA}^{\mathrm{TM}}\right.$ ) assay measures cytokeratin 19 gene expression in whole LNs. We recently showed that

Electronic supplementary material The online version of this article (https://doi.org/10.1245/s10434-019-07971-y) contains supplementary material, which is available to authorized users.

(C) The Author(s) 2019

First Received: 31 January 2019;

Published Online: 13 November 2019

H. Yamamoto, MD, PhD

e-mail: hyamamoto@sahs.med.osaka-u.ac.jp
$17.6 \%$ of pN0 stage II CRC patients were OSNA positive, suggesting a correlation between OSNA results and disease recurrence. This multicenter, prospective study investigateed the prognostic value of the OSNA assay for pStage II CRC patients.

Methods. We examined 204 CRC patients who were preoperatively diagnosed as $\mathrm{cN} 0$ and $\mathrm{cN} 1$ and surgically treated at 11 medical institutions across Japan. Nine patients were excluded, and 195 patients (Stage I: $n=50$, Stage II: $n=70$, Stage III: $n=75$ ) were examined. All LNs, harvested from patients, were examined histopathologically using one-slice hematoxylin-eosin staining. Furthermore, half of the LNs was examined by the OSNA assay. Patients were classified according to the UICC staging criteria and OSNA results, and the 3-year, diseasefree survival (DFS) of each cohort was analyzed. 
Results. Average $21.2 \mathrm{LNs} /$ patient were subject to pathological examination. Approximately half of all harvested LNs (average, 9.4 LNs/patient) were suitable for the OSNA assay. Significantly lower 3-year DFS rates were observed in pStage (pathological Stage) II OSNA-positive patients than in OSNA-negative patients $(p=0.005)$. Among all assessed clinical and pathological parameters, only the OSNA result significantly affected 3-year DFS rates in pStage II CRC patients $(p=0.027)$.

Conclusions. This study shows that OSNA positivity is a risk factor for recurrence of the patients with pStage II CRC.

Patients with stages I-II (pN0) colorectal cancer (CRC) with no high-risk factors are usually treated by surgical resection and avoid adjuvant chemotherapy, which is only recommended for patients with high-risk stage II and stage III disease, due to significant side effects. ${ }^{1,2}$ The standard histopathological evaluation of lymph nodes (LNs) in patients with CRC is based on hematoxylin and eosin (H\&E) staining of LNs and usually involves only approximately $5 \%$ of $\mathrm{LN}$ tissues (2-5- $\mu$ m-thick $\mathrm{LN}$ sections). Therefore, the sensitivity of the LN analysis often is low, leading to disease recurrence in up to $25 \%$ of patients with pN0 disease. ${ }^{3-6}$

Accumulating evidence supports the usefulness of molecular methods for identifying micrometastases and enhancing the sensitivity of pathological cancer staging. ${ }^{7}$ One of these molecular methods, the one-step nucleic acid amplification $\left(\mathrm{OSNA}^{\mathrm{TM}}\right.$ ) assay, was first introduced by Tsujimoto et al. $^{8}$ for detecting LN metastases in patients with breast cancer. The authors demonstrated a $98.2 \%$ agreement rate between OSNA results and routine histopathological findings from the same $\mathrm{LN}$, with practically no false-positive results. Subsequent studies have further demonstrated the high sensitivity of OSNA in detecting LN metastasis of breast, gastric, lung, and col-

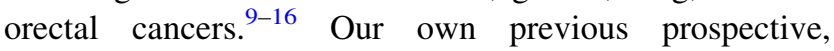
multicenter studies conducted in Japan demonstrated the usefulness of the OSNA assay as a complementary tool for diagnosing LN metastasis and CRC staging. ${ }^{17,18}$ The later study revealed that $17.6 \%$ (13/74) of patients with histologically node-negative stage II CRC were nodal positive by the OSNA assay. ${ }^{18}$ Based on this observation, we hypothesized that positive results of the OSNA assay in patients with node-negative pStage II CRC would correlate with poor disease prognosis. To investigate the prognostic value of the OSNA assay in patients with pStage II CRC, we conducted this multicenter trial.

\section{MATERIALS AND METHODS}

\section{Patients}

This prospective, multicenter study conducted in Japan was approved by the institutional review board of Tokyo Women's Medical University (approval number: 4267). The University Medical Information Network of Japan registration number of the study is UMIN000023233. In addition, ethical approval was obtained from each participating institution. Informed consent was obtained from all patients before enrollment, and patients who were treated with neoadjuvant therapy before surgery, had distant metastases, or had any previous or current cancer were excluded.

A total of 204 patients with CRC who were preoperatively diagnosed as having $\mathrm{cN} 0$ and $\mathrm{cN} 1$ cancer using a computed tomography or magnetic resonance imaging scan and who were surgically treated at 11 medical institutions across Japan between May 2012 and August 2013 were recruited for our study. An open complete mesocolic excision or laparoscopic surgery was performed for tumor resection. LNs were harvested from tissue specimens immediately after surgery using a standardized study protocol. ${ }^{19}$ The Union for International Cancer Control classification was used for patient staging. Four patients with pStage IV CRC, four with follow-up durations $<50$ days, and one with no follow-up data were excluded. Among the remaining 195 patients, 50 were diagnosed with pStage I CRC, 70 with pStage II CRC, and 75 with pStage III CRC.

\section{Study Design}

This study was designed to assess the clinical significance of OSNA positivity in patients with pStage II CRC, and survival rates of 70 patients with pStage II CRC were analyzed. The flow chart of the study design is shown in Fig. 1. The decision to administer adjuvant treatment was solely based on the results of the standard histopathological examination and not on the OSNA results.

\section{LN Processing and Examination}

Immediately after surgery, LNs were harvested from fresh biopsy specimens. When the long axis was $\geq 4 \mathrm{~mm}$, LNs were divided into two pieces, and one piece was used for the OSNA assay and the other for standard histology (one slice H\&E staining). Small LNs that did not have sufficient tissue to divide into two pieces (i.e., $<4 \mathrm{~mm}$ ) were only examined by histopathology. Thus, all of the LNs were examined by standard histology (average 21.2, median 19.0 LNs/patient, range 1-75), and average 9.4, 
FIG. 1 Flowchart of this study's experimental design

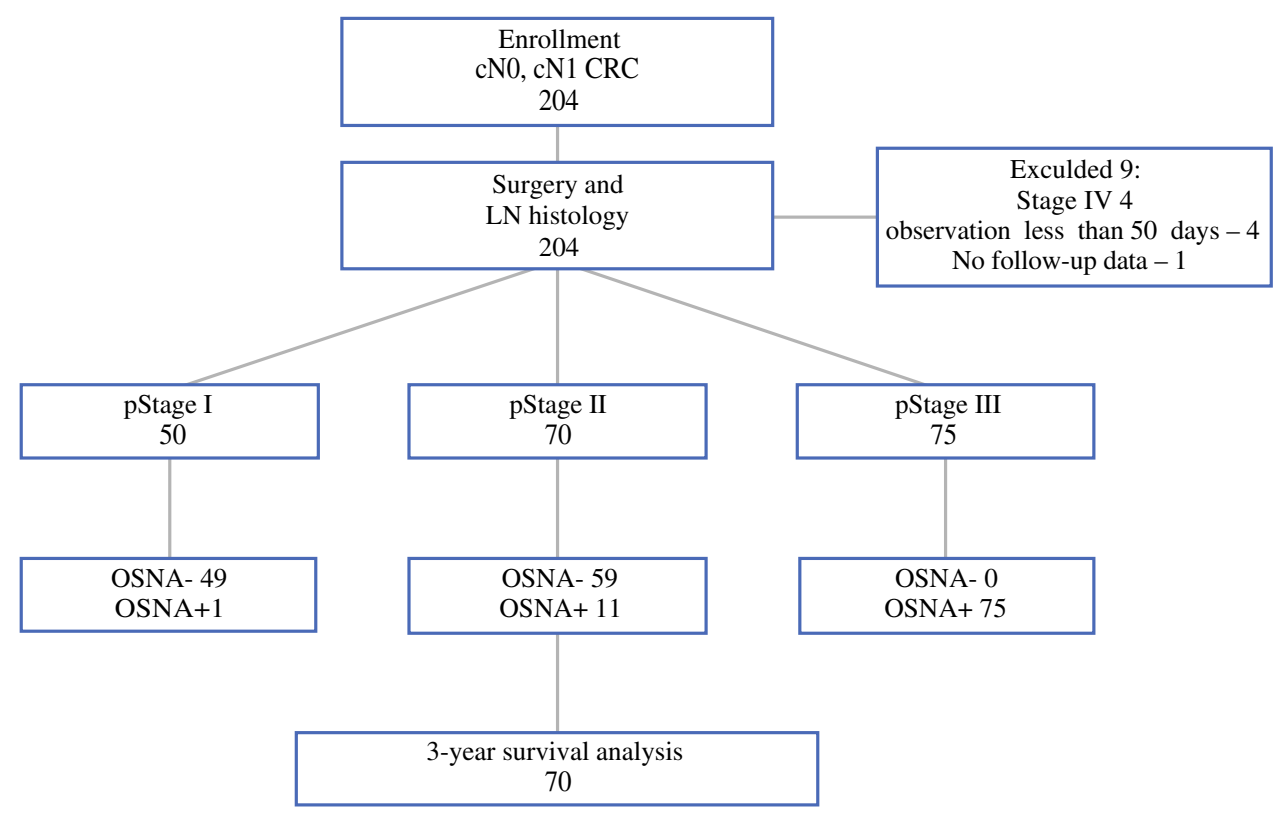

median 8.0 LNs/patient (range 2-25) were examined by the OSNA assay. The LN tissues used for OSNA assays were frozen and stored at $-80{ }^{\circ} \mathrm{C}$ until the assay was performed.

\section{OSNA Assay}

The OSNA assay has been previously described. ${ }^{17,18}$ Briefly, LNs were homogenized with $4 \mathrm{~mL}$ of a lysis buffer solution (Lynorhag; Sysmex, Kobe, Japan) after surgically removing the extranodal tissue and centrifuged at $10,000 \times g$. A $2-\mu \mathrm{L}$ sample of the supernatant was analyzed using the RD-100i system (Sysmex), which is an automated gene amplification detection system, using a reverse transcription loop-mediated isothermal amplification method and with the Lynoamp BC kit (Sysmex). Pyrophosphate, a byproduct of the reaction, indicated the degree of amplification. The precipitation of magnesium pyrophosphate caused a change in turbidity, correlating with the CK19 mRNA copy number in the original lysate. A preestablished standard curve of three calibrators that contained different CK19 mRNA copy numbers was used in the assay to calculate CK19 mRNA copy numbers. For each assay, standard positive and negative control samples were used for quality assurance. LNs containing $>250 \mathrm{mRNA}$ copies/ $\mu \mathrm{L}$ were considered OSNA positive as previously described. ${ }^{17}$

\section{Statistical Analysis}

Postoperative overall survival (OS) and disease-free survival (DFS) curves were produced using the KaplanMeier method. OS was measured from the date of surgery to the date of death or the last follow-up examination. To evaluate the significance of differences in survival rates among the groups, a log-rank test was performed. A univariate analysis with the Cox proportional hazards model was used to establish associations between OSNA assay results and other histopathological parameters. All statistical comparisons were conducted using the $\chi^{2}$ test. $P$ values $<0.05$ were considered statistically significant. All statistical analyses were conducted using Prism Mac version 5.0b (graphpad.com) and JMP Pro version 13.1.0 (SAS Institute Inc.) software.

\section{RESULTS}

\section{OSNA-Based Upstaging}

When OSNA assay results were combined with TNM standard histopathological staging (single-section $\mathrm{H} \& \mathrm{E}$ staining) for 204 patients with $\mathrm{cNO}$ and $\mathrm{cN} 1 \mathrm{CRC}$, most of

TABLE 1 Staging by histopathological examinations with OSNA evaluations

\begin{tabular}{lrrrrl}
\hline & \multicolumn{3}{l}{ OSNA + pathology } & \multirow{2}{*}{ Upstage rate $\%$} \\
\cline { 2 - 4 } & I & II & III & Total & \\
\hline Pathology & & & & & \\
I & 49 & 0 & 1 & 50 & 2.0 \\
II & 0 & 59 & 11 & 70 & 15.7 \\
III & 0 & 0 & 75 & 75 & - \\
Total & 49 & 59 & 87 & 195 & - \\
\hline
\end{tabular}


the upstaging occurred for patients with pStage II, of whom 11 of 70 patients $(15.7 \%)$ were OSNA positive (Table 1). OSNA-positive LNs were found mostly in the paracolic or pararectal LNs near the primary tumor in 10 of 11 OSNApositive Stage II CRC patients. A comparison of demographic and histopathological characteristics of patients who were OSNA negative and OSNA positive is shown in Supplementary Table 1 . For 22 of 70 patients $(31 \%)$, the number of harvested LNs was $<12$. Postoperative adjuvant chemotherapy (Supplementary Table 2) was performed for 19 patients $(27 \%)$ based only on histological data and the principal physician's decision (OSNA-positive group 55\%, OSNA negative group $22 \%$ ). The median follow-up period was 48.5 months (range: 1.7-53.4 months).

\section{DFS and OS of OSNA-Negative and OSNA-Positive Cohorts in pStage II CRC}

In pStage II CRC, significantly higher 3-year DFS rates were observed in OSNA-negative patients than in OSNApositive patients ( $86 \%$ vs. $55 \% ; p=0.005$; Fig. 2a), whereas 3-year OS did not significantly differ between OSNA-negative and OSNA-positive patients $(p=0.914$; Fig. 2b).

\section{Univariate Analysis of OSNA Assay Results}

A univariate analysis of OSNA positivity confirmed its significance as a recurrence risk factor for patients with pStage II CRC ( $p=0.027$; Table 2). Other demographic and histopathological parameters, including age, sex, tumor location, tumor size, vascular and lymphatic invasion, and other features, were not risk factors for tumor recurrence in patients with pStage II CRC (Table 2).

The histopathological characteristics of five recurrent tumors in OSNA-positive patients are presented in Table 3. The site of metastases and number of OSNA mRNA copies

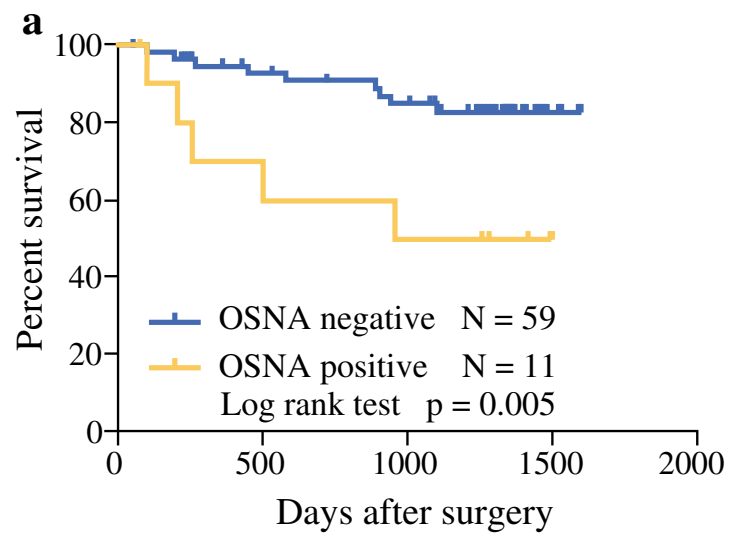

per microliter varied. No associations were observed among recurrent site, primary tumor wall invasion depth, and primary tumor site. Standard adjuvant chemotherapy with oral anticancer agents was administered to four of five patients, and only one patient with OSNA-positive recurrence had $<12$ LNs resected and analyzed.

\section{DISCUSSION}

The prediction of tumor recurrence after resection in patients with pStage II CRC is challenging. ${ }^{20,21}$ This prospective, multicenter study confirmed previous observations of the presence of undetected nodal micrometastases that were identified by the OSNA assay in patients with pStage II CRC and demonstrated that nodal positivity in the OSNA assay was an objective risk factor in these patients. $^{22,23} \mathrm{We}$ found that $15.7 \%$ of pStage II CRC patients were OSNA-positive. Other studies also showed that pNO CRC patients were up-staged by OSNA test in $15.3-28.8 \% .^{13,15,24,25}$ It is suggested that difference of detection rate depends on sampling of $\mathrm{LN}$ volume and number. ${ }^{24}$ Notably Rakislova et al. ${ }^{24}$ reported that $20.8 \%$ pNO cases were OSNA-positive by the pooling method which would be helpful as compared to time consuming and expensive individual analysis of LNs.

Previous similar studies were unable to assess the prognostic value of the molecular detection of CK19 mRNA copies in LNs of patients with pStage II CRC because of the limited 2-year observation time. ${ }^{26}$ In this study, we clearly demonstrated the correlation between molecular LN status and 3-year DFS rates. Furthermore, we showed that the 3 -year DFS of patients with positive OSNA results was significantly lower than that of those with negative OSNA results. However, no difference in OS was found between these groups, most likely because OS assessment requires a longer follow-up period and larger sample of patients who are OSNA-positive.

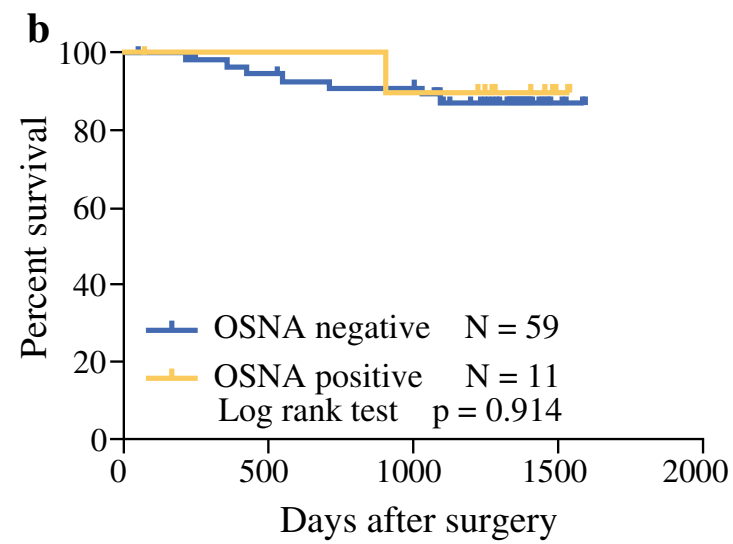

FIG. 2 Survival rates of OSNA-positive and OSNA-negative patients in pStage II CRC patients. a 3-year disease-free survival rate and b 3-year overall survival rate 
TABLE 2 Univariate analysis of the OSNA results

\begin{tabular}{|c|c|c|c|}
\hline & Hazard ratio & $95 \% \mathrm{CI}$ & $p$ value \\
\hline OSNA status in LNs $[(+)$ vs. $(-)]$ & 3.856 & $1.184-11.178$ & 0.027 \\
\hline Age $(>70$ vs. $\leq 70$ years $)$ & 1.711 & $0.594-5.202$ & 0.317 \\
\hline Sex (male vs. female) & 1.774 & $0.613-5.773$ & 0.294 \\
\hline Tumor location (rectum vs. colon) & 1.737 & $0.393-5.569$ & 0.423 \\
\hline Colon tumor location (left vs. right) & 2.238 & $0.675-8.556$ & 0.189 \\
\hline Tumor size $(\leq 4.5$ vs. $>4.5 \mathrm{~cm})$ & 1.22 & $0.424-3.706$ & 0.712 \\
\hline Differentiation (well ${ }^{\mathrm{a}}$, moderate ${ }^{\mathrm{b}}$ vs. poor ${ }^{\mathrm{c}}$, muc $^{\mathrm{d}}$, others) & $5.50 \mathrm{E}+08$ & $0.555-0.555$ & 0.138 \\
\hline Lymphatic invasion (present vs. absent) & 1.954 & $0.675-6.360$ & 0.219 \\
\hline Venous invasion (present vs. absent) & 1.008 & $0.350-3.061$ & 0.989 \\
\hline $\mathrm{T}$ stage (T4 vs. T3) & 2.302 & $0.631-6.885$ & 0.188 \\
\hline Retrieved lymph node number ( $<12$ vs. $\geq 12$ ) & 2.161 & $0.709-6.239$ & 0.167 \\
\hline
\end{tabular}

${ }^{\mathrm{a}}$ Well indicates a well-differentiated adenocarcinoma

${ }^{\mathrm{b}}$ Moderate indicates a moderately differentiated adenocarcinoma

${ }^{\mathrm{c}}$ Poor indicates a poorly differentiated adenocarcinoma

${ }^{\mathrm{d}}$ Muc indicates a mucinous adenocarcinoma

TABLE 3 Recurrences in patients with OSNA-positive pStage II CRC

\begin{tabular}{|c|c|c|c|c|c|c|c|c|}
\hline & Recurrence & PT & Location & $\begin{array}{l}\text { Number of harvested } \\
\text { LNs }\end{array}$ & $\begin{array}{l}\text { OSNA (copies/ } \\
\text { uL) }\end{array}$ & Histology & & Adjuvant chemotherapy \\
\hline $\begin{array}{c}\text { Case } \\
1\end{array}$ & Lung & $\mathrm{T} 3$ & $\mathrm{~S}$ & 20 & 350 & Moderate* & Yes & $\begin{array}{l}\text { Tegafur/uracil plus leucovorin (UFT/ } \\
\text { LV) }\end{array}$ \\
\hline $\begin{array}{c}\text { Case } \\
2\end{array}$ & Liver & T3 & RS & 9 & 1600 & Moderate & Yes & $\begin{array}{l}\text { Tegafur/uracil plus leucovorin (UFT/ } \\
\text { LV) }\end{array}$ \\
\hline $\begin{array}{c}\text { Case } \\
3\end{array}$ & Lung & $\mathrm{T} 3$ & A & 25 & 8600 & Moderate & Yes & $\begin{array}{l}\text { Tegafur/uracil plus leucovorin (UFT/ } \\
\text { LV) }\end{array}$ \\
\hline $\begin{array}{c}\text { Case } \\
4\end{array}$ & Local & $\mathrm{T} 4 \mathrm{~b}$ & $\mathrm{~T}$ & 26 & 2500 & Moderate & No & - \\
\hline $\begin{array}{c}\text { Case } \\
5\end{array}$ & Lung & T3 & A & 14 & 1100 & Moderate & Yes & Tegafur/gimeracil/teracil (S-1) \\
\hline
\end{tabular}

*Moderate indicates a moderately differentiated adenocarcinoma

Yamamoto et al. ${ }^{27}$ reported that the 5-year OS and DFS of patients with higher CEA (carcinoembryonic antigen) mRNA expression levels, top $30 \%$ of micrometastases in pStage II CRC, were significantly low, and the volume of micrometastases was related to the prognosis of the pStage II CRC. Compared with the findings of Yamamoto et al.'s study, only $15.7 \%$ of pStage II CRC were molecular positive in OSNA assay in our study. The OSNA assay can discriminate macrometastases from micrometastases by assessing metastasis volume. ${ }^{8}$ Therefore, metastasis volume of OSNA positivity in our study would be much higher than that in Yamamoto et al.'s study, demonstrating that the OSNA status is related to the DFS of pStage II CRC.

According to the univariate analysis, cancer recurrence correlated only with the OSNA status $(p=0.027)$, while other parameters were not significant in this study. The guidelines of ASCO, ESMO, or NCCN recommend different risk factors for stage II CRC among the parameters, such as vascular invasion, lymphatic invasion, T4, obstruction, perforation, $<12$ LNs examined, poorly or undifferentiated histology, high serum CEA and others, but the evidence level of these factors is not high. ${ }^{28-30}$ Considering that lymph node metastasis is a well-established predictive marker for survival in stage III CRC patients, it is likely that high metastasis volume detected by OSNA test may predict pStage II patients' survival as well.

Recently Wild et al. ${ }^{31}$ performed a systematic review and meta-analysis of OSNA use in CRC and concluded that long-term outcomes and the value of adjuvant therapy in those upstaged by OSNA should be clarified before routine use of OSNA test. In this regard, our study may be constructive because it firstly showed significantly worse 3-year DFS in OSNA-positive pStage II group. However, 
we should emphasize that this study has limitation of small sample size, which may be associated with relatively high relapse rate of stage II patient compared with the largescale study. ${ }^{32}$ Another limitation is that 22 of 70 stage II (31.4\%) CRC patients had $<12$ LNs examined. We cannot deny a possibility that a portion of them might be at stage III if $\geq 12$ LNs had been examined although resected LN numbers were not significantly different between OSNA(+) and OSNA(-) group in pStage II CRC patients.

Because the OSNA test is at present covered by insurance in Japan for diagnosis of LN metastasis of CRC, cumulative data in clinics would lead to firm conclusion in near future. It is well established that the presence of LN metastasis is a clear indication for adjuvant chemotherapy after cancer resection. ${ }^{30}$ Our results support the idea that patients with pNO OSNA-positive CRC might also need chemotherapy after curative surgery. To achieve this goal, we are currently underway to conduct the observational research in which the recurrence rate will be compared between the groups of no treatment or adjuvant chemotherapy after surgery both in OSNA-positive pStage II CRC patients. The result would clarify whether adjuvant chemotherapy is beneficial to patients with OSNA-positive pStage II CRC.

\section{CONCLUSIONS}

This prospective, multicenter study is the first to assess the prognostic value of OSNA positivity in patients with pStage II CRC, which serves as a marker of high recurrence risk. Further studies are warranted to optimize treatment strategies in this setting.

ACKNOWLEDGMENT Editorial support based on authors' detailed directions in the form of medical writing, assembling tables, creating high-resolution images, collating author comments, copyediting, fact checking, and referencing was provided by Editage, Cactus Communications, and the conduct of the research and preparation of the article were funded by Sysmex Corporation.

FUNDING This work was supported by Sysmex Corporation.

DISCLOSURE Michio Itabashi received grants from Chugai Pharmaceutical Co., Takeda Pharmaceutical Co., Taiho Pharmaceutical Co., Pfizer Inc., Astellas Pharma Inc. form April 2017 to March 2018.

OPEN ACCESS This article is distributed under the terms of the Creative Commons Attribution 4.0 International License (http://crea tivecommons.org/licenses/by/4.0/), which permits unrestricted use, distribution, and reproduction in any medium, provided you give appropriate credit to the original author(s) and the source, provide a link to the Creative Commons license, and indicate if changes were made.

\section{REFERENCES}

1. Compton CC. Optimal pathologic staging: defining stage II disease. Clin Cancer Res. 2007;13:S6862-70.

2. Labianca R, Nordlinger B, Beretta GD, et al. ESMO Guidelines Working Group. Early colon cancer: ESMO Clinical Practice Guidelines for diagnosis, treatment and follow-up. Ann Oncol. 2013;24 Suppl 6:vi64-72.

3. Choi HK, Law WL, Poon JT. The optimal number of lymph nodes examined in stage II colorectal cancer and its impact of on outcomes. BMC Cancer. 2010;10:267.

4. Maguire A, Sheahan K. Controversies in the pathological assessment of colorectal cancer. World $J$ Gastroenterol. 2014;20:9850-61.

5. Meyer J, Cohen S, Ruth K, Sigurdson E, Hall M. Young age increases risk of lymph node positivity in early-stage rectal cancer. J Natl Cancer Inst. 2016;108:djv284.

6. Resch A, Langner C. Lymph node staging in colorectal cancer: old controversies and recent advances. World J Gastroenterol. 2013;19:8515-626.

7. Sirop S, Kanaan M, Korant A, et al. Detection and prognostic impact of micrometastasis in colorectal cancer. J Surg Oncol. 2011;103:534-7.

8. Tsujimoto M, Nakabayashi K, Yoshidome K, et al. One-step nucleic acid amplification for intraoperative detection of lymph node metastasis in breast cancer patients. Clin Cancer Res. 2007;13:4807-16.

9. Hunter-Smith AE, Rayter Z. One-step nucleic acid amplification: the possible value in assessing sentinel lymph node metastasis during mastectomy. Breast Cancer. 2018;10:13-21.

10. Kumagai K, Yamamoto N, Miyashiro I, et al. Multicenter study evaluating the clinical performance of the OSNA assay for the molecular detection of lymph node metastases in gastric cancer patients. Gastric Cancer. 2014;17:273-80.

11. Hayama M, Chida M, Karube Y, et al. One-step nucleic acid amplification for detection of lymph node metastasis in lung cancer. Ann Thorac Cardiovasc Surg. 2014;20:181-4.

12. Croner RS, Schellerer V, Demund H, et al. One step nucleic acid amplification (OSNA) - a new method for lymph node staging in colorectal carcinomas. J Transl Med. 2010;8:83.

13. Güller U, Zettl A, Worni M, et al. Molecular investigation of lymph nodes in colon cancer patients using one-step nucleic acid amplification (OSNA): a new road to better staging? Cancer. 2012;118:6039-45.

14. Yamamoto N, Daito M, Hiyama K, et al. An optimal mRNA marker for OSNA (One-step nucleic acid amplification) based lymph node metastasis detection in colorectal cancer patients. Jpn J Clin Oncol. 2013;43:264-70.

15. Croner RS, Geppert CI, Bader FG, et al. Molecular staging of lymph node-negative colon carcinomas by one-step nucleic acid amplification (OSNA) results in upstaging of a quarter of patients in a prospective, European, multicentre study. $\mathrm{Br} J$ Cancer. 2014; 110:2544-50.

16. Vogelaar FJ, Reimers MS, van der Linden RL, et al. The diagnostic value of one-step nucleic acid amplification (OSNA) for sentinel lymph nodes in colon cancer patients. Ann Surg Oncol. 2014;21:3924-30.

17. Yamamoto H, Sekimoto M, Oya M, et al. OSNA-based novel molecular testing for lymph node metastases in colorectal cancer patients: results from a multicenter clinical performance study in Japan. Ann Surg Oncol. 2011;18:1891-8.

18. Yamamoto $\mathrm{H}$, Tomita $\mathrm{N}$, Inomata $\mathrm{M}$, et al. OSNA-assisted molecular staging in colorectal cancer: a prospective multicenter trial in Japan. Ann Surg Oncol. 2016;23:391-6. 
19. O'Connor ES, Greenblatt DY, LoConte NK, et al. Adjuvant chemotherapy for stage II colon cancer with poor prognostic features. J Clin Oncol. 2011;29:3381-8.

20. Quah HM, Chou JF, Gonen M, et al. Identification of patients with high-risk stage II colon cancer for adjuvant therapy. Dis Colon Rectum. 2008;51:503-7.

21. Sloothaak DA, Sahami S, van der Zaag-Loonen HJ, et al. The prognostic value of micrometastases and isolated tumour cells in histologically negative lymph nodes of patients with colorectal cancer: a systematic review and meta-analysis. Eur J Surg Oncol. 2014;40:263-9.

22. Rahbari NN, Bork U, Motschall E, et al. Molecular detection of tumor cells in regional lymph nodes is associated with disease recurrence and poor survival in node-negative colorectal cancer: a systematic review and meta-analysis. $J$ Clin Oncol. 2012;30:60-70.

23. Aldecoa I, Atares B, Tarragona J, et al. Molecularly determined total tumour load in lymph nodes of stage I-II colon cancer patients correlates with high-risk factors. A multicentre prospective study. Virchows Arch. 2016;469:385-94.

24. Rakislova N, Montironi C, Aldecoa I, et al. Lymph node pooling: a feasible and efficient method of lymph node molecular staging in colorectal carcinoma. J Transl Med. 2017;15:14.

25. Brito MJ, Honavar M, Cipriano MA, et al. Molecular staging of patients with colon cancer. The C-Closer-II study: a multicentre study in Portugal. Acta Med Port. 2018;31:661-9.

26. Märkl B, Rößle J, Arnholdt HM, et al. The clinical significance of lymph node size in colon cancer. Mod Pathol. 2012;25:1413-22.
27. Yamamoto H, Murata K, Fukunaga M, et al. Micrometastasis volume in lymph nodes determines disease recurrence rate of stage II colorectal cancer: a prospective multicenter trial. Clin Cancer Res. 2016;22:3201-8.

28. Benson AB III, Schrag D, Somerfield MR, et al. American society of clinical oncology recommendations on adjuvant chemotherapy for stage II colon cancer. J Clin Oncol. 2004;22:3408-19.

29. Van Cutsem E, Oliveira J. Primary colon cancer: ESMO clinical recommendations for diagnosis, adjuvant treatment and followup. Ann Oncol. 2009;20:49-50.

30. NCCN. Colon cancer. National comprehensive cancer network. 2019. https://www.nccn.org/professionals/physician_gls/pdf/colo n.pdf.

31. Wild JB, Iqbal N, Francombe J, Papettas T, Sanders DS, Ramcharan S. Is it time for one-step nucleic acid amplification (OSNA) in colorectal cancer? A systematic review and metaanalysis. Tech Coloproctol 2017;21:693-9.

32. Matsuda $\mathrm{C}$, Ishiguro $\mathrm{M}$, Teramukai $\mathrm{S}$, et al. A randomised-controlled trial of 1-year adjuvant chemotherapy with oral tegafururacil versus surgery alone in stage II colon cancer: SACURA trial. Eur J Cancer. 2018;96:54-63.

Publisher's Note Springer Nature remains neutral with regard to jurisdictional claims in published maps and institutional affiliations. 